\title{
Fibroblast heterogeneity: implications for human disease
}

\author{
Magnus D. Lynch ${ }^{1,2}$ and Fiona M. Watt ${ }^{1}$ \\ 1King's College London Centre for Stem Cells and Regenerative Medicine, Guy's Hospital, Great Maze Pond, London, United Kingdom. ${ }^{2}$ St John's Institute of Dermatology, King's College London, London, \\ United Kingdom.
}

\begin{abstract}
Fibroblasts synthesize the extracellular matrix of connective tissue and play an essential role in maintaining the structural integrity of most tissues. Researchers have long suspected that fibroblasts exhibit functional specialization according to their organ of origin, body site, and spatial location. In recent years, a number of approaches have revealed the existence of fibroblast subtypes in mice. Here, we discuss fibroblast heterogeneity with a focus on the mammalian dermis, which has proven an accessible and tractable system for the dissection of these relationships. We begin by considering differences in fibroblast identity according to anatomical site of origin. Subsequently, we discuss new results relating to the existence of multiple fibroblast subtypes within the mouse dermis. We consider the developmental origin of fibroblasts and how this influences heterogeneity and lineage restriction. We discuss the mechanisms by which fibroblast heterogeneity arises, including intrinsic specification by transcriptional regulatory networks and epigenetic factors in combination with extrinsic effects of the spatial context within tissue. Finally, we discuss how fibroblast heterogeneity may provide insights into pathological states including wound healing, fibrotic diseases, and aging. Our evolving understanding suggests that ex vivo expansion or in vivo inhibition of specific fibroblast subtypes may have important therapeutic applications.
\end{abstract}

\section{Introduction}

A paradigm of the metazoan body plan is the combination of epithelial and mesenchymal elements into structured three-dimensional organs. Fibroblasts are cells that synthesize and integrate structural proteins such as collagen and elastin into the extracellular matrix (ECM) of most mesenchymal tissues (1-4). It has long been believed that fibroblasts exhibit differing functional identities (5), and in recent years, evidence has emerged to support this concept. Fibroblasts are characterized by the expression of markers including vimentin and procollagen I $\alpha 2$ chain (1). However, these markers are not considered specific for fibroblasts (6), and it has not been demonstrated that they are expressed on all fibroblast types.

Fibroblast heterogeneity can be considered according to stage of development, tissue of origin, or tissue microenvironment. The mammalian skin has proven a particularly tractable tissue in which to address these questions. The epidermis, a stratified squamous epithelium, overlies the dermis, a mesenchymal tissue (Figure 1). In addition to fibroblasts and ECM components, the dermis, in common with other mesenchymal tissues, incorporates a wide range of other cell types, including blood vessel components (endothelial cells, smooth muscle cells, and blood vessel-associated fibroblasts) (7), eccrine and apocrine sweat glands, lymphatic vessel components, neurons (8), sensory receptors, and tissue-res-

Conflict of interest: F.M. Watt is a non-executive director of the Cell and Gene Therapy Catapult Ltd, a member of the Stem Cell Advisory Board of Frequency Therapeutics Inc., and a member of the OxStem Advisory Board.

Reference information: / Clin Invest. 2018;128(1):26-35.

https://doi.org/10.1172/JCI93555 ident immune cells $(9,10)$. These other cell types are sufficiently numerous that fibroblasts represent a minority cell population in the adult dermis.

A simple definition of cellular identity is that it represents the set of macromolecules present within a cell at a specific point in time. However, this definition omits the possibility that cells with identical gene expression patterns at a particular time point can respond differently to an external stimulus or differentiate spontaneously because of epigenetic or other factors. A further complication is that spatial location with respect to ECM components and other cells may play a role in the maintenance of a specific cellular identity, and these signals can be lost when cells are extracted from the tissue. As a consequence of these factors, the dissection of cellular identity and lineage relationships is inherently more challenging for cells that constitute solid tissues than for cells of the hemopoietic system.

Here, we discuss emerging concepts relating to cellular identity and lineage relationships of fibroblasts. We begin by discussing fibroblast heterogeneity according to tissue and anatomical site of origin. Subsequently, we examine evidence in support of the concept that individual tissues, such as the dermis, comprise multiple fibroblast subtypes. We consider to what extent differences are specified intrinsically by transcriptional regulatory networks and epigenetic mechanisms versus by extrinsic factors that depend on the spatial context within the tissue, such as secreted factors, cellcell communication, and interactions with the ECM.

While full details of the mechanisms governing fibroblast cellular identity remain to be determined, it is likely that specific fibroblast subtypes will play key roles in wound healing, in diseases characterized by excessive fibrosis, in cancer stroma, and 


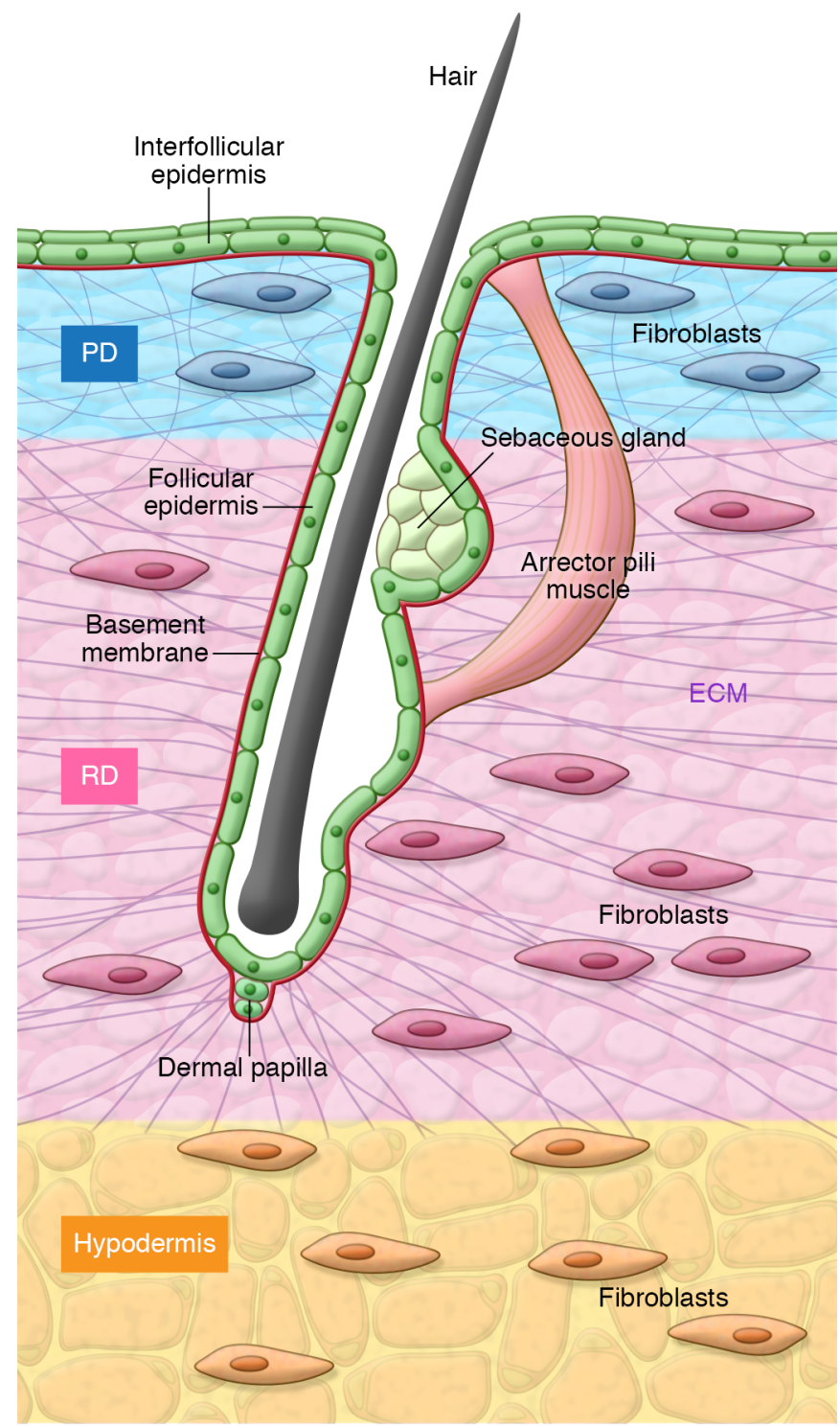

potentially in tissue aging. This suggests the possibility that in vivo inhibition of specific fibroblast subtypes or cellular therapy consisting of ex vivo expansion and subsequent reintroduction may have therapeutic benefit in the treatment of such conditions.

\section{Fibroblast heterogeneity according to anatomical origin}

Mesenchymal-derived connective tissues including heart (11), lung (12), gastrointestinal tract (13), and muscle (14) contain fibroblasts that fulfill specialized functions (15). Differences in gene expression have been demonstrated between dermal and nondermal fibroblasts $(16,17)$, and fibroblasts derived from different anatomical sites have differing developmental origins, including the neural crest, lateral plate mesoderm, and dermatomyotome (18-20). Moreover, there are significant differences in the architecture of the dermis at different body sites, which are associated with a differing potential for disease processes such as the formation of keloid scars (21). Differences in morphology and behavior in cultured fibroblasts derived from different body sites
Figure 1. Structure of murine dorsal skin. The skin has two layers, the epidermis and the dermis. The epidermis is a stratified squamous epithelium with associated adnexal structures such as hair follicles and sebaceous glands. It is separated from the underlying connective tissue, the dermis, by a basement membrane (red). The three dermal layers are the papillary dermis $(P D)$, reticular dermis $(R D)$, and hypodermis/white adipose tissue. The dermal papilla and arrector pili muscle constitute two specialized populations of dermal mesenchymal cells. Adapted with permission from Science (143)

(15) are correlated with differing patterns of Hox gene expression that reflect the developmental origin of the cells $(16,17)$. Therefore, differences in the behavior of fibroblasts from different body sites likely reflect a combination of intrinsic differences and the role of factors such as mechanical stress that differs between body regions (22).

Fibroblasts derived from the bone marrow, which are the cells that adhere when plated in culture, are worthy of special consideration. This population of fibroblasts have the capacity to differentiate into multiple lineages (23) and have been termed mesenchymal stem or stromal cells (MSCs). Initial reports suggested that MSCs can contribute to the dermis (24-26), but subsequent studies do not appear to support this finding (27-29). Interestingly, clinical trials have suggested that the infusion of MSCs into the blood may ameliorate several diseases, such as graft-versus-host disease $(30,31)$. However, there has been no demonstration that these MSCs stably engraft, and it has been proposed that any beneficial effects are achieved by release of paracrine factors (32).

Recent results support the concept that pericytes - blood vessel-associated fibroblasts - are the in vivo counterpart of MSCs (33). Pericytes are associated with and encircle vascular channels in most tissues $(34,35)$. A number of markers have been proposed for the identification of pericytes, including NG2 (35) and RGS5 (36). It remains unclear whether different subtypes of pericytes are associated with arteries, veins, and lymphatics. Within muscle, it appears that pericytes have the capability to differentiate to muscle fibers (37), but at the present time there is no evidence that pericytes have the capability to differentiate into other dermal lineages. A specific subset of ADAM12-expressing perivascular fibroblasts present in skin and muscle are activated by wounding and contribute to collagen overproduction in the scar (38). Genetic ablation of these cells reduces collagen production and scarring (38).

Cardiac fibroblasts constitute the largest cellular population within the heart (11) and are located throughout the myocardium, interspersed between myocardiocytes (11). They exhibit specific expression of discoidin domain receptor 2 (DDR2) (39) and play a critical role in the regulation of normal myocardial function in addition to the pathological remodeling that accompanies hypertension and heart failure. It has long been recognized that the effects of angiotensin II signaling on cardiac fibroblasts and myocytes lead to hypertrophy and fibrosis (11). Proinflammatory signaling by IL- $1 \beta$ and TNF- $\alpha$ also leads to pathological alterations in collagen synthesis by cardiac fibroblasts that accompany myocardial failure (40). Fibroblasts are also integral to the structure of the lung, and, as for the heart, the effects of proinflammatory cytokines such as IL-1 $\alpha$ on pulmonary fibroblasts play an important role in the progression of fibrotic lung disease (41). 
A Human

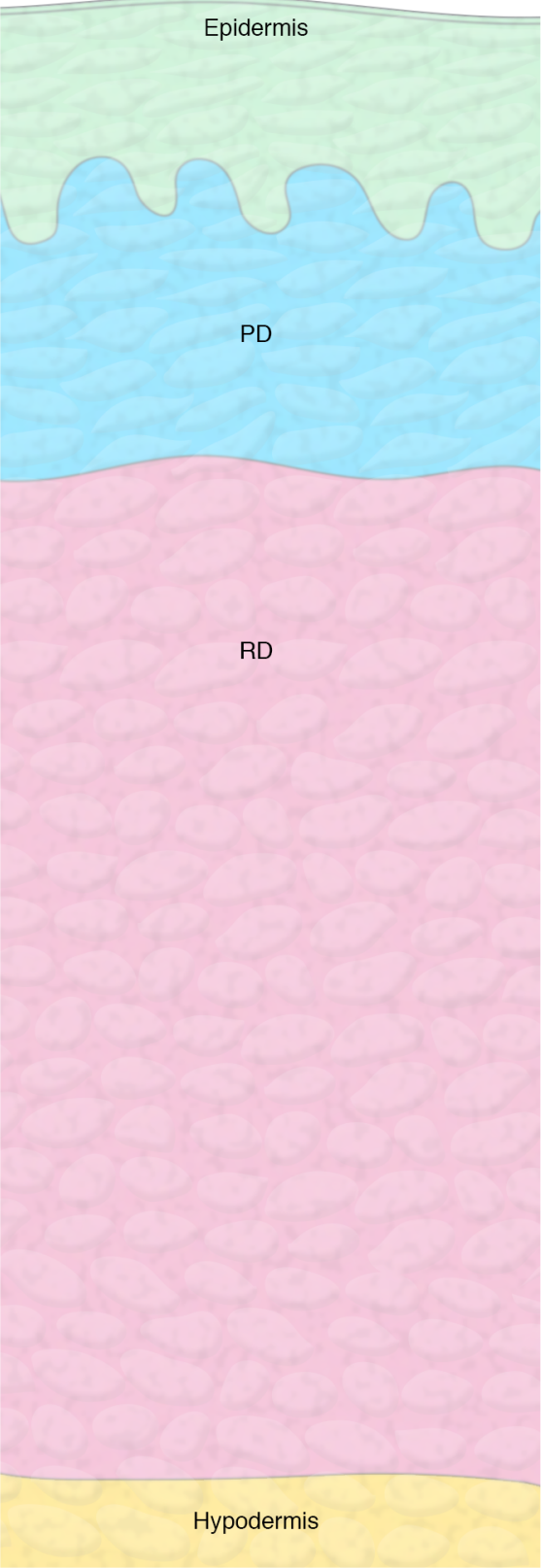

Figure 2. Comparison of human and murine skin. The interfollicular epidermis and dermis are thicker in human than in mouse skin. In most body sites mice possess a higher density of hair follicles. In human skin the boundary between the epidermis and dermis undulates, whereas it is flat in mouse skin. Adapted with permission from Cold Spring Harbor Perspectives in Biology (4).
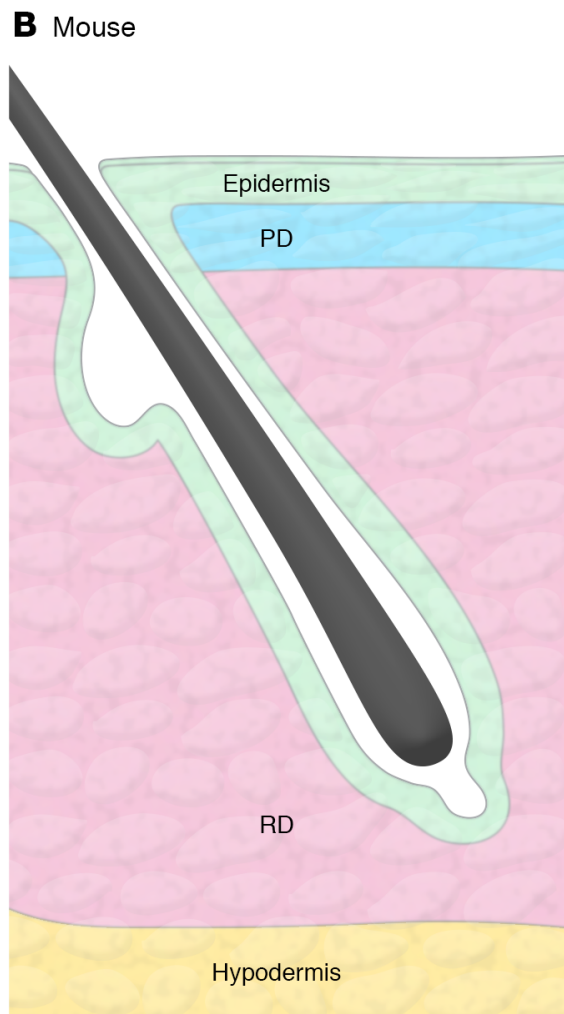

\section{Fibroblast identity differs between papillary and reticular dermis}

The mammalian dermis represents an archetypal mesenchymal tissue largely composed of ECM elements, primarily type I and type III collagens, but also other collagen subtypes, proteoglycans, and elastin (4). It serves as a structural scaffold, and a basement membrane enriched in type IV collagen (44) separates it from the epidermis, a stratified squamous epithelium that forms the outermost layer of the skin. The dermis is penetrated by numerous appendageal structures, including hair follicles and sweat glands, which manifest as specialized invaginations of the epithelium, for which the basement membrane is in continuity with the interfollicular epidermis (Figure 1). The detailed microstructure of the dermis differs between human and mouse skin. In humans, there is a relatively low density of hair follicles on body sites outside of the scalp, with intervening interfollicular epidermis exhibiting invaginations known as rete ridges. In mice, the density of hair is higher and rete ridges are not prominent (Figure 2).

The dermis has distinct layers that are readily identified histologically: the papillary dermis is the superficial layer of the dermis that lies immediately below the epidermis (Figure 1). This is composed of dense but poorly oriented collagen bundles that provide structural support to the overlying basement membrane (4). Beneath this layer is the reticular dermis, which comprises directionally oriented, thicker collagen bundles with intervening spaces $(45,46)$. It has long been recognized that there are differences in the composition of the ECM components at the ultrastructural level that constitute the papillary and reticular dermis. This has led to the hypothesis that ECM composition reflects differences in fibroblast identity in the tissue compartments.

Microarray analysis of cultured fibroblasts derived from mechanically separated dermis has revealed differences in gene expression (47); however, subsequent studies have yet to reproduce proposed markers

Thus far we have considered differences in fibroblasts arising from distinct anatomical sites; however, as we shall discuss, multiple, functionally distinct fibroblast subtypes can arise from a single tissue. This is best understood in the dermis. Although fibroblast heterogeneity in other organs has yet to be investigated in detail, it seems likely that it exists. For example, fibroblasts derived from kidney cortical and medullary regions exhibit differences in cell culture and in their responses to mitogenic stimulation $(42,43)$. of papillary and reticular layers. Dermatome experiments of this nature are inherently limited, since the level of sectioning cannot be precisely controlled, and therefore the possibility that reticular fibroblasts are included in the papillary sample cannot be excluded. Furthermore, since they are studied in the form of explant cultures, it is not clear to what extent in vitro selection for specific cell subpopulations plays a role and to what degree blood vessel-associated fibroblasts, known as pericytes, contribute. 


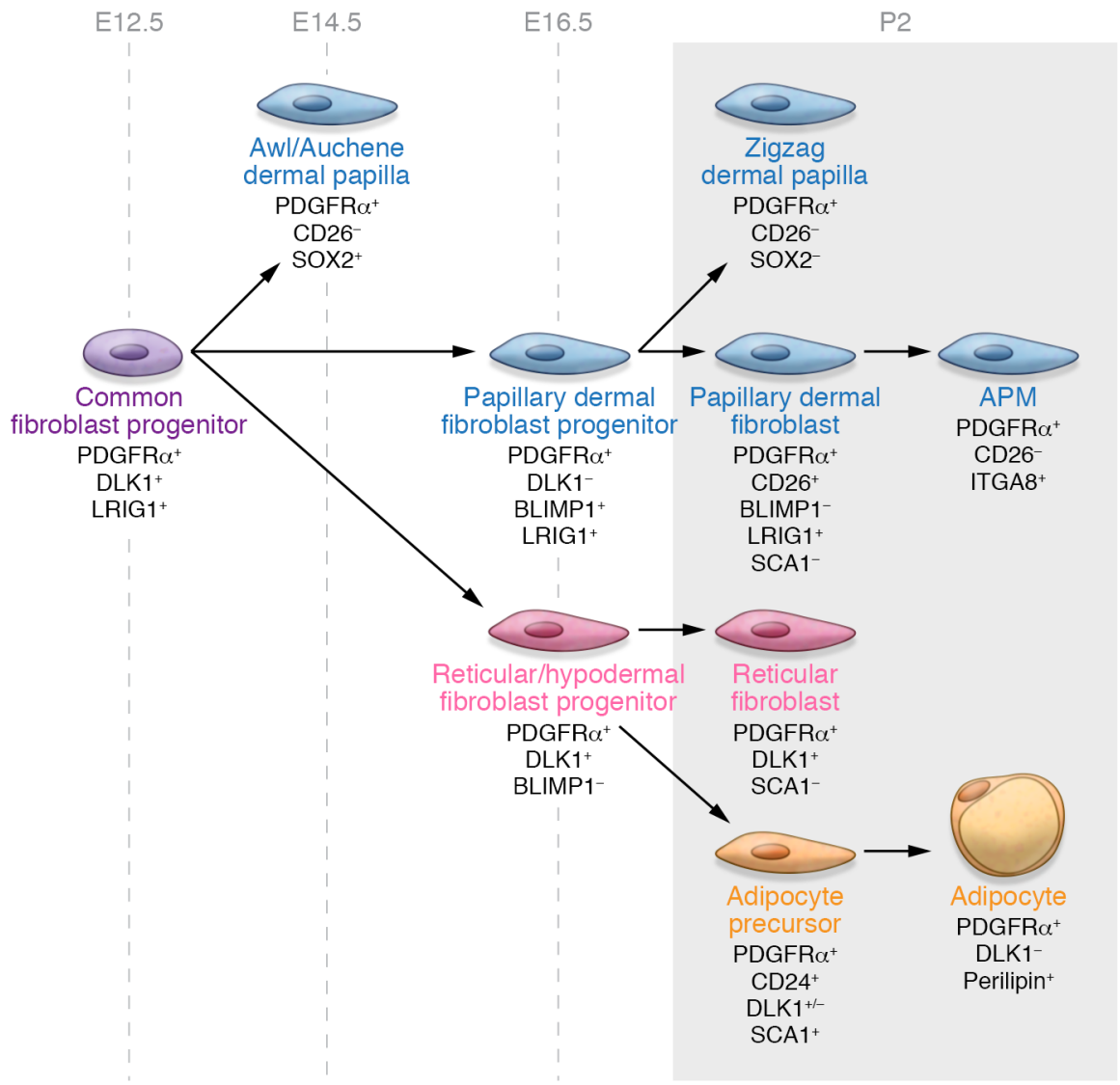

Figure 3. Mouse dermal fibroblast lineages. Dermal fibroblasts derive from common fibroblast progenitor cells and differentiate into specific lineages by postnatal day 2 ( $\mathrm{P} 2)$. These subtypes display distinct functions. For example, papillary fibroblasts are essential in the coordination of the hair cycle and the formation of hair follicles after injury, and reticular fibroblasts mediate early wound repair responses. Adapted with permission from Trends in Cell Biology (6). APM, arrector pili muscle. papillary fibroblasts, reticular fibroblasts, and dermal papillae are derived from a multipotent mesenchymal progenitor that is present at embryonic day 12.5 (E12.5), with lineage commitment occurring by E16.5 (56). At postnatal day 2, papillary, reticular, and hypodermal markers can be differentiated according to the expression of cell surface markers including CD26, SCA1, and DLK1, with CD26 marking the upper dermal lineage (ref. 56 and Figure 3). Papillary fibroblasts are required for new hair follicle formation following wounding, whereas reticular fibroblasts mediate the early events in wound repair and express so-called fibroblast activation markers such as $\alpha$-smooth muscle actin (aSMA) (56).

A subsequent study identified fibroblast subtypes in the mouse dermis according to the expression of Engrailed-1 (En1) and CD26. The authors found that the $\mathrm{CD} 26^{+} \mathrm{En} 1^{+}$population is responsible for dermal development and fibrosis and also contributes to the cancer stroma (57). The differences observed between these two studies with respect to CD26 expression highlight that the expression of lineage markers can be dynamic, varying according to stage of development even where lineage identity is maintained. Further studies are needed to establish whether similar lineages are present in the human dermis.
Immunohistochemical studies have demonstrated differences in the expression of collagen subtypes and proteoglycans between papillary and reticular dermis $(45,48-50)$, although interpretation of these studies is complicated by differences in the density of fibroblasts and ECM elements in different regions of the dermis. Papillary and reticular fibroblasts can be isolated by explant culture of mechanically sectioned (dermatomed) human dermis. Explant fibroblast cultures derived from these layers exhibit differences in a number of parameters, including rate of cell division, contraction, and the expression of various collagens and proteoglycans $(5,51,52)$. Cultured fibroblasts derived from the papillary dermis support the formation of a normal stratified epidermis in three-dimensional organotypic culture more effectively than fibroblasts from the reticular dermis $(47,53,54)$. This may reflect differences in both secreted factors and ECM elements (55); however, as noted above, these observations are potentially confounded by selection inherent in explant cultures and by contamination with other fibroblast subpopulations, including pericytes.

More recently, we and others demonstrated the presence of functional dermal fibroblast subtypes in the mouse dermis. Using PDGFR $\alpha$ as a pan-fibroblast marker, linage tracing experiments revealed that dermal fibroblast populations including

\section{Additional fibroblast subtypes present within the dermis}

Two separate populations of fibroblasts are associated with hair follicles (Figure 1). Hair follicle dermal papilla (FDP) fibroblasts play a central role in the development of hair and the coordination of the hair cycle $(58,59)$. Both primary and cultured FDP fibroblasts have the capability to induce the development of epithelial hair follicle elements at ectopic sites in the skin (60-63). In the mouse, dermal papillae are derived from a common fibroblast progenitor that is present at E12.5 (56). Lineage tracing of Sox2-Cre FDP fibroblasts reveals that these cells have only a minority contribution to wound healing (64). However, there is some evidence that in certain circumstances dermal papilla (FDP) fibroblasts can differentiate to other cell types, including nerve and cartilage (65). A second population of hair follicle-associated fibroblasts, dermal sheath (DS) fibroblasts, encapsulate the hair shaft external to epithelial components of the hair follicle, from which they are separated by a basement membrane termed the glassy membrane.

A number of markers have been described for human and mouse FDP and DS fibroblasts, including CD133 (in human and mouse) and nonspecific alkaline phosphatase (in human) (66-68). Marker expression changes according to stage of the hair cycle (69-72) and, in the mouse, depends on the hair follicle type, with 
Sox2 expressed in all dermal papillae from E16.5 but confined to guard, awl, and auchene hairs from E18.5 (70). Variation has also been observed in the expression of markers for hair follicles according to anatomical site of origin; for example, the expression of androgen receptors is higher in regions of the scalp that are susceptible to male pattern hair loss (73).

Additional populations of fibroblasts within the dermis include pericytes, preadipocytes, and myofibroblasts. As discussed in detail above, pericytes are associated with and encircle vascular channels $(34,35)$. Wound healing in the adult organism is characterized by the formation of an ECM-rich scar that lacks normal cutaneous appendages such as hair and sweat glands (74). Wounding leads initially to the formation of a fibrin-rich plug, followed by the accumulation of leukocytes and the migration of $\alpha \mathrm{SMA}^{+}$myofibroblasts from adjacent dermis and underlying adipose tissue into the wound (75-78). Subsequently, the infiltrating $\alpha \mathrm{SMA}^{+}$fibroblasts develop a contractile phenotype (79). Lineage tracing in the mouse demonstrates that the initial wave of fibroblast migration is derived from lower-lineage (i.e., reticular) fibroblasts (56).

Preadipocytes are fibroblasts with the capacity to differentiate to adipocytes. These are enriched in the lower dermis (80). In humans, they express the CD36 cell surface marker (81). Lineage tracing of preadipocytes with Cre-labeled adiponectin reveals that preadipocytes proliferate and mature adipocytes migrate to contribute to wound repair (82). Preadipocytes are differentiated from other fibroblasts by the expression of ZFP423, a zinc finger transcription factor (83). Peroxisome proliferator-activated receptor gamma 2 (PPAR $\gamma 2)$ is a master transcriptional regulator of the subsequent adipogenic differentiation program (84).

Interestingly, a recent study has demonstrated that myofibroblasts have the capacity to transdifferentiate to adipocytes in response to bone morphogenetic protein (BMP) signals arising from hair follicles in the healing wound (85), suggesting that preadipocytes are not the only cell type that can give rise to mature fat cells in the dermis.

\section{Intrinsic mechanisms specifying fibroblast cellular identity}

Cellular identity is determined both by intrinsic mechanisms, including transcriptional regulatory networks (86) and epigenetic processes, and by extrinsic factors, including cell-cell signaling, soluble signaling mediators, or ECM elements. A key role for intrinsic mechanisms in the specification of fibroblast identity is demonstrated by classic heterotopic transplantation experiments, which show an instructive role of mesenchyme in specifying the identity of overlying epithelial tissues. The culture of dental epithelium from chick embryos overlying dermal mesenchyme led to the development of epidermis including cutaneous appendages (87). Similar results were seen with the ability of wing mesenchyme to induce the development of feathers in heterotopic tissues (88).

Consistent with these findings, fibroblasts derived from palms and soles are able to induce the expression of keratin 9 in nonpalmoplantar keratinocytes, but this is not the case for fibroblasts derived from nonpalmoplantar sites (89). Furthermore, studies of hair transplantation demonstrate that follicles taken from the posterior scalp retain their developmental identity when transplanted to anterior scalp (90), and FDP fibroblasts maintain hair induction capability for both mouse and human, at least for early passages in culture $(71,91,92)$.

In most cases, the details of transcriptional regulatory networks governing specification of fibroblast subtypes are unknown. However, the identity of certain key transcriptional regulators has been established. Sox 2 appears to play a key role in the specification of certain hair follicle types within the mouse dermis. Cre-mediated ablation of Sox 2 in FDP cells leads to a reduction in awl/auchene hairs and an increase in zigzag hairs in mice (93). Conversely, expression of Sox18 is required for the formation of zigzag hairs (94). Interestingly, Sox $2^{+}$FDP fibroblasts maintain their identity in culture (71), consistent with intrinsic differences in cellular identity.

\section{Extrinsic factors governing fibroblast cellular identity}

Extrinsic factors governing cell fate include cell-cell contact, secreted signaling factors, and interactions with ECM elements. The exposure of a particular cell to these influences will depend on spatial context within the tissue. Coculture experiments reveal that keratinocytes instruct fibroblasts to produce a range of cytokines, including IL-6 and FGF7 $(95,96)$. IL-1 has been identified as an inducing factor in these coculture experiments $(96,97)$. Conversely, factors secreted by fibroblasts influence the growth and differentiation of keratinocytes, indicating reciprocal cellcell interaction (98). Additionally, ECM context has an important impact on fibroblast cellular identity. Fibroblasts cultured as a monolayer exhibit differences in collagen expression and morphology in comparison with fibroblasts in three-dimensional culture. There are also differences observed between collagen and fibrin gels (99-101). An instructive role of ECM elements in cellular identity is supported by tissue engineering strategies in which the ECM of decellularized organs is repopulated with cells $(102,103)$. While the full details of these interactions remain to be discovered, it is possible that a dynamic interplay between ECM signaling and production is important in the maintenance of fibroblast cellular identity in tissues.

With regard to specific signaling pathways that are implicated in the regulation of fibroblast cell identity, the emerging picture is of a complex interplay of multiple signaling pathways that synergistically regulate fibroblast identity and function within the dermis. Preeminent among these is the Wnt pathway. Epidermal Wnt signaling is required for hair follicle morphogenesis $(92,104)$. Epidermal overexpression of the Wnt downstream effector $\beta$-catenin in all basal epidermal keratinocytes via the keratin 14 (K14) promoter leads to de novo hair follicle morphogenesis in adult skin; this is accompanied by extensive remodeling of the dermis ECM (105). These effects are mediated by both TGF- $\beta$ and Hedgehog signaling (106) and influence the lower and upper dermal fibroblast lineages, respectively. Pharmacological deletion or genetic ablation of these pathways prevents the response to epidermal Wnt/ $\beta$-catenin overexpression (106).

Other signaling pathways, including secreted BMPs and insulin-like growth factors, play a role in mediating the effects of keratinocyte-specific $\beta$-catenin expression, leading to an increase in adipogenesis. Conversely, inhibition of Wnt/ $\beta$-catenin signal- 
ing in the epidermis impairs adipogenesis (107). Interestingly, it appears that the consequences of Wnt/ $\beta$-catenin are cell type-specific. $\beta$-Catenin stabilization in dermal fibroblasts during development leads to fibrosis and impairs adipogenesis (108). Wnt/ $\beta$-catenin expression in the adult dermis inhibits de novo hair follicle formation in healing wounds, and postnatal ablation of $\beta$-catenin in dermal fibroblasts enhances new hair formation (109).

Additional signaling pathways that have been implicated in the regulation of dermal fibroblasts include the Notch pathway, which has a role in the regulation of inflammation and hair follicle morphogenesis $(110,111)$. TGF- $\beta$ signaling also plays a key role in wound healing and scar formation (3). BMP signaling has been implicated in the regulation of hair follicle morphogenesis (112114). Furthermore, BMP signaling is required for the transdifferentiation of myofibroblasts to adipocytes in healing wounds (85).

Dysregulation of Wnt signaling is observed in a number of conditions that are characterized by aberrant or excessive fibrosis. The profibrotic effects of TGF- $\beta$ are mediated through canonical Wnt signaling via a decreased expression of the Wnt antagonist Dickkopf-1 (115). A role for Wnt signaling has also been seen in renal fibrosis (116), idiopathic pulmonary fibrosis (117), and the increased fibrosis that is observed to accompany muscle regeneration with aging (118).

Fibroblasts play a critical role in maintaining the structural integrity of the dermis. To fulfill this function, there must exist a mechanism for fibroblasts to adapt and respond to mechanical stress. The details of these processes are emerging. YAP signaling via the Hippo pathway has been implicated in the regulation of ECM stiffness (119). Furthermore, a role for focal adhesion kinase (FAK) signaling has been demonstrated in the response of wound repair to mechanical stress (22). FAK signaling is triggered by cutaneous injury and signals through ERK to induce the expression of monocyte chemoattractant protein-1 (MCP-1), a profibrotic chemokine.

\section{Therapeutic implications}

The emerging concept that mammalian tissues, such as the dermis, contain fibroblasts with differing functional identities has profound implications for the understanding of a wide range of pathological states, including diseases characterized by excessive fibrosis such as scarring, scleroderma, cancer, and potentially including chronological aging. It is likely that fibroblast subtypes identified in mice will be conserved in the human, and this is the subject of ongoing investigation.

In contrast to adult mammals, wounds in the fetus heal without scarring $(120,121)$. An understanding of the role of fibroblast subtypes in this process may suggest strategies to reduce scarring in adult organisms. Scar-free healing is observed up until 24 weeks of human gestation and is conserved across many mammalian species (121). Studies examining human fetal skin grafts to immunodeficient mice indicate that this is an intrinsic property of the fetal skin (122), possibly mediated by differences in the fibroblast subtypes present. Interestingly, epidermal activation of canonical Wnt signaling in the basal layer of mouse epidermis leads to remodeling of the adult dermis to a neonatal-like state with formation of new hair follicles (105). Wnt signaling is also required for regeneration of nails and digits in neonatal mice $(29,123)$ and may contribute to differences in wound healing between the fetus and adult.
The involvement of specific fibroblast lineages may have particular relevance to diseases characterized by excessive fibrosis and scarring such as scleroderma, keloids, graft-versus-host disease, and pulmonary fibrosis. Keloidal scaring is characterized by excessive fibroblast proliferation and ECM deposition (124, 125), and keloid-derived fibroblasts exhibit differences in gene expression and behavior in culture compared with fibroblasts from healthy dermis $(126,127)$. It has been proposed that these disease-associated alterations in fibroblast behavior reflect epigenetic alterations in gene expression patterns, and in keeping with this, fibroblasts in rheumatoid arthritis exhibit differences in DNA methylation and histone acetylation (128). Fibroblasts derived from patients with scleroderma exhibited differences in methylation of the FLI1 promoter (129). Epigenetic differences were also observed between keloidal and control fibroblasts (130). Histone deacetylase inhibitors were able to normalize to some extent the differences in gene expression seen with fibroblasts derived from the airways of patients with severe pulmonary hypertension (131).

Epithelial tumors consist of a mixture of epithelial elements, fibroblasts, and ECM components. Cancer-associated fibroblasts (CAFs) play an important role in evolution of solid tumors. There is an extensive crosstalk between CAFs and the epithelial elements of tumors (132), wherein fibroblasts associated with later stages of malignancy exhibit different phenotypes. Interestingly, CAFs exhibit an increased expression of $\alpha \mathrm{SMA}$ (1), which may reflect alterations in the composition of fibroblast subtypes. YAP signaling as a consequence of mechanical stress also plays an important role in remodeling of the ECM that accompanies carcinogenesis. There is evidence for heterogeneity of CAFs (133), but the extent to which these differences reflect fibroblast subtypes present in normal tissue remains to be determined, and further characterization of fibroblast subtypes may increase our understanding of these questions.

Aging is associated with characteristic changes in the structure of the dermis, including dermal thinning and flattening of the dermal-epidermal junction, with loss of the normal rete ridges $(134,135)$. Interestingly, this appears to preferentially affect the papillary dermis: alterations in the behavior of papillary dermal fibroblasts derived from older donors have been observed in three-dimensional cultures (136). Changes are also prominent in other components of connective tissue such as the large blood vessels. In the skin, proliferation of fibroblasts occurs during mouse embryonic development; however, subsequent growth of the animal is not associated with further fibroblast proliferation and is instead achieved simply by an increase in the ECM volume between adjacent fibroblasts (109). This is consistent with the observation that the rate of fibroblast division in the adult dermis is very low in the steady state (137). It may be that during aging this relative paucity of fibroblasts in the adult is insufficient to maintain the structural integrity of the ECM. Alternatively, it may be that there are age-associated alterations in the composition of fibroblast subtypes within the dermis.

A goal of regenerative medicine is the restoration of normal form and function either via cellular therapies in vivo or by the reconstitution of functional organs ex vivo. Efforts to accomplish the latter have led to attempts to reconstitute intact organs via the 
reintroduction of cellular populations to decellularized ECM templates $(102,103)$. This has proven challenging, and future efforts may be informed by a better understanding of the fibroblast subtypes present in these tissues. There have also been attempts to prepare organotypic skin substitutes comprising both fibroblasts and keratinocytes for grafting onto chronic wounds or other sites (138). It is recognized that, in vitro, fibroblasts derived from the upper dermis better support the formation of a structurally and functionally normal epidermis $(47,53,54)$, and it is likely that an understanding of fibroblast subtypes present in human dermis will facilitate the prospective isolation of cells for the repopulation of dermal templates of this nature.

A detailed characterization of fibroblast subtypes in the human in combination with an understanding of the differential contribution of these subtypes to human disease may offer novel strategies for the treatment of diseases. In this context, it could be envisaged that the action of deleterious fibroblast subtypes could be inhibited, perhaps via inhibition of signaling pathways specific to these subtypes or via monoclonal antibody-mediated deletion. In support of this concept, experimentally reducing the number of fibroblasts during wound healing in the mouse can reduce the degree of fibrosis (38). Alternatively, beneficial subtypes may be expanded ex vivo, perhaps via manipulation of cell signaling pathways. Trials of fibroblast cell therapy are already under way for the treatment of poorly healing ulcers (139) and epidermolysis bullosa (140). Notably, papillary fibroblasts appear to be more effective when used in the construction of tissue-engineered skin substitutes (141).

\section{Conclusions and outstanding questions}

The emerging picture from the research outlined in this Review is that fibroblasts represent a family of related cell types with differing functions in the formation and maintenance of ECM in connective tissues and play a key role in coordinating the function of other cell types within the tissue. The understanding of fibroblast heterogeneity is most advanced for the mammalian dermis, because this is a particularly tractable tissue for experimental studies. However, it is probable that these principles are common to other vertebrate tissues including the intestine, lung, heart, and muscle.

Many details of the mechanisms that establish and maintain vertebrate cellular identity remain unclear, and the outstanding questions in the field of fibroblast cell biology reflect this. It is presently unclear to what extent differences in fibroblast cellular identity are specified intrinsically versus reflecting a consequence of spatial context within the tissue such as cell-cell contacts and signaling. Intrinsically specified differences in fibroblast functional identity likely reflect a combination of transcriptional regulatory networks with epigenetic mechanisms. At present, in most cases we do not know the full details of the transcriptional regulatory networks that dictate the identity of fibroblast subtypes.
The importance of extrinsic signals in the maintenance of cellular identity is of particular relevance to the ex vivo expansion of fibroblasts in culture. There is evidence that fibroblasts exhibit a significant shift in the expression of cell surface markers in the transition to cell culture (142). This may reflect an influence of extrinsic signals that are required for the maintenance of fibroblast cell identity, which will be lost on transition to culture. Furthermore, since minimal fibroblast cell division occurs in vivo, it may be that the powerful mitogenic stimuli encountered in cell culture lead to dramatic alterations in cellular identity. These issues are of considerable relevance to efforts to develop fibroblast therapies, particularly as ex vivo strategies will have a higher complexity for regulatory approval and likely a higher cost. Furthermore, they are important for the study of human disease, since many studies have sought to infer disease mechanisms through the study of cultured patient-derived fibroblasts.

Another unresolved question is the degree of plasticity that exists in fibroblast cell identity - can one type of fibroblast differentiate to another given the appropriate stimuli, or is there is a hierarchy of fibroblasts exhibiting progressively restricted differentiation potential? To what extent do mesenchymal stem/stromal cells correspond to fibroblast subtypes present elsewhere in the body, and do they have the capacity to differentiate into other fibroblast subtypes and incorporate into tissues?

It is not clear to what extent fibroblast subtypes identified in the mouse dermis are conserved in the human. To understand the role that fibroblast subtypes play in human disease, markers that permit subtype identification in human histological sections and permit the prospective isolation of these subtypes by flow cytometry will be required. Previous studies of human dermis have attempted to define markers that distinguish fibroblasts cultured separately from the papillary and reticular dermis (47). However, this approach has not yielded cell surface markers that permit the prospective isolation of fibroblast subpopulations directly from the skin. These issues are areas of active investigation, and it is likely that recent advancements in the area of single-cell-based transcriptional and epigenetic analysis will catalyze a greater understanding.

\section{Acknowledgments}

We gratefully acknowledge financial support from the Academy of Medical Sciences (to MDL); and from the Wellcome TrustMedical Research Council and Cancer Research UK (to FMW).

Address correspondence to: Fiona M. Watt, Centre for Stem Cells and Regenerative Medicine, King's College London, 28th Floor, Tower Wing, Guy's Campus, Great Maze Pond, London SE1 9RT, United Kingdom. Phone: 044.207.188.5608; E-mail: fiona.watt@ kcl.ac.uk.
1. Kalluri R, Zeisberg M. Fibroblasts in cancer.

Nat Rev Cancer. 2006;6(5):392-401.

2. Parsonage $\mathrm{G}$, et al. A stromal address code defined by fibroblasts. Trends Immunol. 2005;26(3):150-156.

3. Tomasek JJ, Gabbiani G, Hinz B, Chaponnier C, Brown RA. Myofibroblasts and mechano-regu- lation of connective tissue remodelling. Nat Rev Mol Cell Biol. 2002;3(5):349-363.

4. Watt FM, Fujiwara H. Cell-extracellular matrix interactions in normal and diseased skin. Cold Spring Harb Perspect Biol. 2011;3(4):a005124.

5. Harper RA, Grove G. Human skin fibroblasts derived from papillary and reticular dermis: differences in growth potential in vitro. Science. 1979;204(4392):526-527.

6. Driskell RR, Watt FM. Understanding fibroblast heterogeneity in the skin. Trends Cell Biol. 2015;25(2):92-99.

7. Detmar M. Molecular regulation of angiogenesis in the skin. JInvest Dermatol.1996;106(2):207-208. 
8. Ansel JC, Kaynard AH, Armstrong CA, Olerud J, Bunnett N, Payan D. Skin-nervous system interactions. J Invest Dermatol. 1996;106(1):198-204.

9. Nestle FO, Nickoloff BJ. A fresh morphological and functional look at dermal dendritic cells. JCutan Pathol. 1995;22(5):385-393.

10. Lugović L, Lipozenocić J, Jakić-Razumović J. Atopic dermatitis: immunophenotyping of inflammatory cells in skin lesions. Int J Dermatol. 2001;40(8):489-494.

11. Camelliti P, Borg TK, Kohl P. Structural and functional characterisation of cardiac fibroblasts. Cardiovasc Res. 2005;65(1):40-51.

12. Ramos C, et al. Fibroblasts from idiopathic pulmonary fibrosis and normal lungs differ in growth rate, apoptosis, and tissue inhibitor of metalloproteinases expression. Am J Respir Cell Mol Biol. 2001;24(5):591-598.

13. Pascal RR, Kaye GI, Lane N. Colonic pericryptal fibroblast sheath: replication, migration, and cytodifferentiation of a mesenchymal cell system in adult tissue. I. Autoradiographic studies of normal rabbit colon. Gastroenterology. 1968;54(5):835-851.

14. Kühl U, Ocalan M, Timpl R, Mayne R, Hay E, von der Mark K. Role of muscle fibroblasts in the deposition of type-IV collagen in the basal lamina of myotubes. Differentiation. 1984;28(2):164-172.

15. Castor CW, Prince RK, Dorstewitz EL. Characteristics of human "fibroblasts" cultivated in vitro from different anatomical sites. Lab Invest. 1962;11:703-713.

16. Rinn JL, Bondre C, Gladstone HB, Brown PO, Chang HY. Anatomic demarcation by positional variation in fibroblast gene expression programs. PLoS Genet. 2006;2(7):e119.

17. Rinn JL, et al. A dermal HOX transcriptional program regulates site-specific epidermal fate. Genes Dev. 2008;22(3):303-307.

18. Houzelstein D, Chéraud Y, Auda-Boucher G, Fontaine-Pérus J, Robert B. The expression of the homeobox gene Msx1 reveals two populations of dermal progenitor cells originating from the somites. Development. 2000;127(10):2155-2164.

19. Le Lièvre CS, Le Douarin NM. Mesenchymal derivatives of the neural crest: analysis of chimaeric quail and chick embryos. J Embryol Exp Morphol. 1975;34(1):125-154.

20. Sriram G, Bigliardi PL, Bigliardi-Qi M. Fibroblast heterogeneity and its implications for engineering organotypic skin models in vitro. Eur JCell Biol. 2015;94(11):483-512.

21. Bayat A, Arscott G, Ollier WE, Ferguson MW, Mc Grouther DA. Description of site-specific morphology of keloid phenotypes in an Afrocaribbean population. Br J Plast Surg. 2004;57(2):122-133.

22. Wong VW, et al. Focal adhesion kinase links mechanical force to skin fibrosis via inflammatory signaling. Nat Med. 2011;18(1):148-152.

23. Minguell JJ, Erices A, Conget P. Mesenchymal stem cells. Exp Biol Med. 2001;226(6):507-520.

24. Badiavas EV, Abedi M, Butmarc J, Falanga V, Quesenberry P. Participation of bone marrow derived cells in cutaneous wound healing. JCell Physiol. 2003;196(2):245-250.

25. Ishii $\mathrm{M}$, et al. Molecular markers distinguish bone marrow mesenchymal stem cells from fibroblasts. Biochem Biophys Res Commun.
2005;332(1):297-303.

26. Kataoka K, et al. Participation of adult mouse bone marrow cells in reconstitution of skin. Am J Pathol. 2003;163(4):1227-1231.

27. Barisic-Dujmovic T, Boban I, Clark SH. Fibroblasts/myofibroblasts that participate in cutaneous wound healing are not derived from circulating progenitor cells. J Cell Physiol. 2010;222(3):703-712.

28. Higashiyama R, et al. Differential contribution of dermal resident and bone marrow-derived cells to collagen production during wound healing and fibrogenesis in mice. J Invest Dermatol. 2011;131(2):529-536.

29. Rinkevich Y, Lindau P, Ueno H, Longaker MT, Weissman IL. Germ-layer and lineage-restricted stem/progenitors regenerate the mouse digit tip. Nature. 2011;476(7361):409-413.

30. von Bonin M, et al. Treatment of refractory acute GVHD with third-party MSC expanded in platelet lysate-containing medium. Bone Marrow Transplant. 2009;43(3):245-251.

31. Resnick IB, et al. Treatment of severe steroid resistant acute GVHD with mesenchymal stromal cells (MSC). Am J Blood Res. 2013;3(3):225-238.

32. Hocking AM, Gibran NS. Mesenchymal stem cells: paracrine signaling and differentiation during cutaneous wound repair. Exp Cell Res. 2010;316(14):2213-2219.

33. Crisan M, et al. A perivascular origin for mesenchymal stem cells in multiple human organs. Cell Stem Cell. 2008;3(3):301-313.

34. Hall AP. Review of the pericyte during angiogenesis and its role in cancer and diabetic retinopathy. Toxicol Pathol. 2006;34(6):763-775.

35. Paquet-Fifield S, et al. A role for pericytes as microenvironmental regulators of human skin tissue regeneration. JClin Invest. 2009;119(9):2795-2806.

36. Cho H, Kozasa T, Bondjers C, Betsholtz C, Kehrl JH. Pericyte-specific expression of Rgs5: implications for PDGF and EDG receptor signaling during vascular maturation. FASEB J. 2003;17(3):440-442.

37. Dellavalle A, et al. Pericytes resident in postnatal skeletal muscle differentiate into muscle fibres and generate satellite cells. Nat Commun 2011;2:499.

38. Dulauroy S, Di Carlo SE, Langa F, Eberl G, Peduto $\mathrm{L}$. Lineage tracing and genetic ablation of ADAM12(+) perivascular cells identify a major source of profibrotic cells during acute tissue injury. Nat Med. 2012;18(8):1262-1270.

39. Goldsmith EC, et al. Organization of fibroblasts in the heart. Dev Dyn. 2004;230(4):787-794

40. Siwik DA, Chang DL, Colucci WS. Interleukin-1beta and tumor necrosis factor- $\alpha$ decrease collagen synthesis and increase matrix metalloproteinase activity in cardiac fibroblasts in vitro. Circ Res. 2000;86(12):1259-1265.

41. Suwara MI, et al. IL- $1 \alpha$ released from damaged epithelial cells is sufficient and essential to trigger inflammatory responses in human lung fibroblasts. Mucosal Immunol. 2014;7(3):684-693.

42. Rodemann HP, Muller GA, Knecht A, Norman JT, Fine LG. Fibroblasts of rabbit kidney in culture. I. Characterization and identification of cell-specific markers. Am J Physiol. 1991;261(2 pt 2):F283-F291.
43. Knecht A, et al. Fibroblasts of rabbit kidney in culture. II. Paracrine stimulation of papillary fibroblasts by PDGF. Am J Physiol. 1991; 261(2 pt 2):F292-F299.

44. Has C, Nyström A. Epidermal basement membrane in health and disease. Curr Top Membr. 2015;76:117-170.

45. Sorrell JM, Caplan AI. Fibroblast heterogeneity: more than skin deep. JCell Sci. 2004; 117(pt 5):667-675.

46. Collins CA, Kretzschmar K, Watt FM. Reprogramming adult dermis to a neonatal state through epidermal activation of $\beta$-catenin. Development. 2011;138(23):5189-5199.

47. Janson DG, Saintigny G, van Adrichem A, Mahé C, El Ghalbzouri A. Different gene expression patterns in human papillary and reticular fibroblasts. J Invest Dermatol. 2012;132(11):2565-2572.

48. Meigel WN, Gay S, Weber L. Dermal architecture and collagen type distribution. Arch Dermatol Res. 1977;259(1):1-10.

49. Zimmermann DR, Dours-Zimmermann MT, Schubert M, Bruckner-Tuderman L. Versican is expressed in the proliferating zone in the epidermis and in association with the elastic network of the dermis. J Cell Biol. 1994;124(5):817-825.

50. Sorrell JM, Carrino DA, Baber MA, Asselineau D, Caplan AI. A monoclonal antibody which recognizes a glycosaminoglycan epitope in both dermatan sulfate and chondroitin sulfate proteoglycans of human skin. Histochem J. 1999;31(8):549-558.

51. Schafer IA, Pandy M, Ferguson R, Davis BR. Comparative observation of fibroblasts derived from the papillary and reticular dermis of infants and adults: growth kinetics, packing density at confluence and surface morphology. Mech Ageing Dev. 1985;31(3):275-293.

52. Akagi A, Tajima S, Ishibashi A, Yamaguchi N, Nagai Y. Expression of type XVI collagen in human skin fibroblasts: enhanced expression in fibrotic skin diseases. J Invest Dermatol. 1999;113(2):246-250.

53. Sorrell JM, Baber MA, Caplan AI. Site-matched papillary and reticular human dermal fibroblasts differ in their release of specific growth factors/ cytokines and in their interaction with keratinocytes. J Cell Physiol. 2004;200(1):134-145.

54. Lee DY, Cho KH. The effects of epidermal keratinocytes and dermal fibroblasts on the formation of cutaneous basement membrane in threedimensional culture systems. Arch Dermatol Res. 2005;296(7):296-302.

55. Varkey M, Ding J, Tredget EE. Differential collagen-glycosaminoglycan matrix remodeling by superficial and deep dermal fibroblasts: potential therapeutic targets for hypertrophic scar. Biomaterials. 2011;32(30):7581-7591.

56. Driskell RR, et al. Distinct fibroblast lineages determine dermal architecture in skin development and repair. Nature. 2013;504(7479):277-281.

57. Rinkevich Y, et al. Skin fibrosis. Identification and isolation of a dermal lineage with intrinsic fibrogenic potential. Science. 2015;348(6232):aaa2151.

58. Sennett R, Rendl M. Mesenchymal-epithelial interactions during hair follicle morphogenesis and cycling. Semin Cell Dev Biol. 2012;23(8):917-927.

59. Driskell RR, Clavel C, Rendl M, Watt FM. Hair 
follicle dermal papilla cells at a glance. J Cell Sci. 2011;124(pt 8):1179-1182.

60. Jahoda CA. Induction of follicle formation and hair growth by vibrissa dermal papillae implanted into rat ear wounds: vibrissa-type fibres are specified. Development. 1992;115(4):1103-1109.

61. Oliver RF. The experimental induction of whisker growth in the hooded rat by implantation of dermal papillae. J Embryol Exp Morphol. 1967;18(1):43-51.

62. Jahoda CA, Horne KA, Oliver RF. Induction of hair growth by implantation of cultured dermal papilla cells. Nature. 1984;311(5986):560-562.

63. Reynolds AJ, Lawrence C, Cserhalmi-Friedman PB, Christiano AM, Jahoda CA. Trans-gender induction of hair follicles. Nature. 1999;402(6757):33-34.

64. Biernaskie J, et al. SKPs derive from hair follicle precursors and exhibit properties of adult dermal stem cells. Cell Stem Cell. 2009;5(6):610-623.

65. Toma JG, et al. Isolation of multipotent adult stem cells from the dermis of mammalian skin. Nat Cell Biol. 2001;3(9):778-784.

66. Anan T, Sonoda T, Asada Y, Kurata S, Takayasu S. Protease-activated receptor-1 (thrombin receptor) is expressed in mesenchymal portions of human hair follicle. J Invest Dermatol. 2003;121(4):669-673.

67. Johnson PL, Butcher EO, Bevelander G. The distribution of alkaline phosphatase in the cyclic growth of the rat hair follicle. Anat Rec. 1945;93:355-358.

68. Ito Y, Hamazaki TS, Ohnuma K, Tamaki K, Asashima M, Okochi H. Isolation of murine hair-inducing cells using the cell surface marker prominin-1/CD133. J Invest Dermatol. 2007;127(5):1052-1060.

69. Rendl M, Lewis L, Fuchs E. Molecular dissection of mesenchymal-epithelial interactions in the hair follicle. PLoS Biol. 2005;3(11):e331.

70. Driskell RR, Giangreco A, Jensen KB, Mulder KW, Watt FM. Sox2-positive dermal papilla cells specify hair follicle type in mammalian epidermis. Development. 2009;136(16):2815-2823.

71. Driskell RR, Juneja VR, Connelly JT, Kretzschmar K, Tan DW, Watt FM. Clonal growth of dermal papilla cells in hydrogels reveals intrinsic differences between Sox2-positive and -negative cells in vitro and in vivo. J Invest Dermatol. 2012;132(4):1084-1093.

72. Enshell-Seijffers D, Lindon C, Morgan BA. The serine protease Corin is a novel modifier of the Agouti pathway. Development. 2008;135(2):217-225.

73. Hibberts NA, Howell AE, Randall VA. Balding hair follicle dermal papilla cells contain higher levels of androgen receptors than those from non-balding scalp. JEndocrinol. 1998;156(1):59-65.

74. Gurtner GC, Werner S, Barrandon Y, Longaker MT. Wound repair and regeneration. Nature. 2008;453(7193):314-321.

75. Rossio-Pasquier P, Casanova D, Jomard A, Démarchez M. Wound healing of human skin transplanted onto the nude mouse after a superficial excisional injury: human dermal reconstruction is achieved in several steps by two different fibroblast subpopulations. Arch Dermatol Res. 1999;291(11):591-599.

76. van den Bogaerdt AJ, van Zuijlen PP, van Galen
M, Lamme EN, Middelkoop E. The suitability of cells from different tissues for use in tissue-engineered skin substitutes. Arch Dermatol Res. 2002;294(3):135-142.

77. Gabbiani G, Ryan GB, Majne G. Presence of modified fibroblasts in granulation tissue and their possible role in wound contraction. Experientia. 1971;27(5):549-550.

78. Gabbiani G. The myofibroblast in wound healing and fibrocontractive diseases. J Pathol. 2003;200(4):500-503.

79. Germain L, Jean A, Auger FA, Garrel DR. Human wound healing fibroblasts have greater contractile properties than dermal fibroblasts. J Surg Res. 1994;57(2):268-273.

80. Festa E, et al. Adipocyte lineage cells contribute to the skin stem cell niche to drive hair cycling. Cell. 2011;146(5):761-771.

81. Abumrad NA, el-Maghrabi MR, Amri EZ, Lopez E, Grimaldi PA. Cloning of a rat adipocyte membrane protein implicated in binding or transport of longchain fatty acids that is induced during preadipocyte differentiation. Homology with human CD36. JBiol Chem. 1993;268(24):17665-17668.

82. Schmidt BA, Horsley V. Intradermal adipocytes mediate fibroblast recruitment during skin wound healing. Development. 2013;140(7):1517-1527.

83. Gupta RK, et al. Transcriptional control of preadipocyte determination by Zfp 423 . Nature. 2010;464(7288):619-623.

84. Tontonoz P, Hu E, Spiegelman BM. Stimulation of adipogenesis in fibroblasts by PPAR gamma 2, a lipid-activated transcription factor. Cell. 1994;79(7):1147-1156.

85. Plikus MV, et al. Regeneration of fat cells from myofibroblasts during wound healing. Science. 2017;355(6326):748-752.

86. Davidson EH, Erwin DH. Gene regulatory networks and the evolution of animal body plans. Science. 2006;311(5762):796-800.

87. Kollar EJ. Tissue interactions in development of teeth and related ectodermal derivatives. Dev $\operatorname{Biol}(N Y)$. 1986;4:297-313.

88. Dhouailly D, Rogers GE, Sengel P. The specification of feather and scale protein synthesis in epidermal-dermal recombinations. Dev Biol. 1978;65(1):58-68

89. Yamaguchi Y, Itami S, Tarutani M, Hosokawa K, Miura H, Yoshikawa K. Regulation of keratin 9 in nonpalmoplantar keratinocytes by palmoplantar fibroblasts through epithelial-mesenchymal interactions. J Invest Dermatol. 1999;112(4):483-488.

90. Orentreich N. Autografts in alopecias and other selected dermatological conditions. Ann N Y Acad Sci. 1959;83:463-479.

91. Horne KA, Jahoda CA, Oliver RF. Whisker growth induced by implantation of cultured vibrissa dermal papilla cells in the adult rat. J Embryol Exp Morphol. 1986;97:111-124.

92. Kishimoto J, Burgeson RE, Morgan BA. Wnt signaling maintains the hair-inducing activity of the dermal papilla. Genes Dev. 2000;14(10):1181-1185

93. Lesko MH, Driskell RR, Kretzschmar K, Goldie SJ, Watt FM. Sox 2 modulates the function of two distinct cell lineages in mouse skin. Dev Biol. 2013;382(1):15-26.

94. James K, Hosking B, Gardner J, Muscat GE, Koopman P. Sox18 mutations in the ragged mouse alleles ragged-like and opossum. Genesis. 2003;36(1):1-6.

95. Werner S, Krieg T, Smola H. Keratinocyte-fibroblast interactions in wound healing. JInvest Dermatol. 2007;127(5):998-1008.

96. Maas-Szabowski N, Shimotoyodome A, Fusenig NE. Keratinocyte growth regulation in fibroblast cocultures via a double paracrine mechanism. JCell Sci. 1999;112(pt 12):1843-1853.

97. Maas-Szabowski N, Stark HJ, Fusenig NE. Keratinocyte growth regulation in defined organotypic cultures through IL-1-induced keratinocyte growth factor expression in resting fibroblasts. J Invest Dermatol. 2000;114(6):1075-1084.

98. Florin L, Maas-Szabowski N, Werner S, Szabowski A, Angel P. Increased keratinocyte proliferation by JUN-dependent expression of PTN and SDF-1 in fibroblasts. J Cell Sci. 2005;118(pt 9):1981-1989.

99. Eckes B, Mauch C, Hüppe G, Krieg T. Downregulation of collagen synthesis in fibroblasts within three-dimensional collagen lattices involves transcriptional and posttranscriptional mechanisms. FEBS Lett. 1993;318(2):129-133.

100. Kessler D, et al. Fibroblasts in mechanically stressed collagen lattices assume a "synthetic" phenotype. JBiol Chem. 2001;276(39):36575-36585.

101.Fluck J, Querfeld C, Cremer A, Niland S, Krieg T, Sollberg S. Normal human primary fibroblasts undergo apoptosis in three-dimensional contractile collagen gels. J Invest Dermatol. 1998;110(2):153-157.

102. Ross EA, et al. Embryonic stem cells proliferate and differentiate when seeded into kidney scaffolds. J Am Soc Nephrol. 2009;20(11):2338-2347.

103. Baptista PM, Siddiqui MM, Lozier G, Rodriguez SR, Atala A, Soker S. The use of whole organ decellularization for the generation of a vascularized liver organoid. Hepatology. 2011;53(2):604-617.

104. Myung PS, Takeo M, Ito M, Atit RP. Epithelial Wnt ligand secretion is required for adult hair follicle growth and regeneration. J Invest Dermatol. 2013;133(1):31-41.

105. Silva-Vargas V, et al. $\beta$-Catenin and Hedgehog signal strength can specify number and location of hair follicles in adult epidermis without recruitment of bulge stem cells. Dev Cell. 2005;9(1):121-131.

106. Lichtenberger BM, Mastrogiannaki M, Watt FM. Epidermal $\beta$-catenin activation remodels the dermis via paracrine signalling to distinct fibroblast lineages. Nat Commun. 2016;7:10537.

107. Donati $G$, et al. Epidermal Wnt $/ \beta$-catenin signaling regulates adipocyte differentiation via secretion of adipogenic factors. Proc Natl Acad Sci U S A. 2014;111(15):E1501-E1509.

108. Mastrogiannaki M, Lichtenberger BM, Reimer A, Collins CA, Driskell RR, Watt FM. $\beta$-Catenin stabilization in skin fibroblasts causes fibrotic lesions by preventing adipocyte differentiation of the reticular dermis. J Invest Dermatol. 2016;136(6):1130-1142.

109. Rognoni $\mathrm{E}$, et al. Inhibition of $\beta$-catenin signalling in dermal fibroblasts enhances hair follicle regeneration during wound healing. Development. 2016;143(14):2522-2535.

110. Hu B, et al. Control of hair follicle cell fate by underlying mesenchyme through a CSLWnt5a-FoxN1 regulatory axis. Genes Dev. 
2010;24(14):1519-1532.

111. Hu B, et al. Multifocal epithelial tumors and field cancerization from loss of mesenchymal CSL signaling. Cell. 2012;149(6):1207-1220.

112. Clavel C, et al. Sox 2 in the dermal papilla niche controls hair growth by fine-tuning BMP signaling in differentiating hair shaft progenitors. Dev Cell. 2012;23(5):981-994.

113. Plikus MV, et al. Self-organizing and stochastic behaviors during the regeneration of hair stem cells. Science. 2011;332(6029):586-589.

114. Sharov AA, et al. Bone morphogenetic protein signaling regulates the size of hair follicles and modulates the expression of cell cycleassociated genes. Proc Natl Acad Sci U S A. 2006;103(48):18166-18171.

115. Akhmetshina A, et al. Activation of canonical Wnt signalling is required for TGF- $\beta$-mediated fibrosis. Nat Commun. 2012;3:735.

116. He W, Dai C, Li Y, Zeng G, Monga SP, Liu Y. Wnt/ $\beta$-catenin signaling promotes renal interstitial fibrosis. J Am Soc Nephrol. 2009;20(4):765-776.

117. Chilosi M, et al. Aberrant Wnt/ $\beta$-catenin pathway activation in idiopathic pulmonary fibrosis. Am J Pathol. 2003;162(5):1495-1502.

118. Brack AS, et al. Increased Wnt signaling during aging alters muscle stem cell fate and increases fibrosis. Science. 2007;317(5839):807-810.

119. Calvo F, et al. Mechanotransduction and YAPdependent matrix remodelling is required for the generation and maintenance of cancer-associated fibroblasts. Nat Cell Biol. 2013;15(6):637-646.

120. Rowlatt U. Intrauterine wound healing in a 20 week human fetus. Virchows Arch A Pathol Anat Histol. 1979;381(3):353-361.

121. Colwell AS, Longaker MT, Lorenz HP. Fetal wound healing. Front Biosci. 2003;8:s1240-s1248.

122.Lorenz HP, Longaker MT, Perkocha LA, Jennings RW, Harrison MR, Adzick NS. Scarless wound repair: a human fetal skin model. Development. 1992;114(1):253-259.
123. Takeo M, et al. Wnt activation in nail epithelium couples nail growth to digit regeneration. Nature. 2013;499(7457):228-232.

124. Jumper N, Paus R, Bayat A. Functional histopathology of keloid disease. Histol Histopathol. 2015;30(9):1033-1057.

125. Lee JY, Yang CC, Chao SC, Wong TW. Histopathological differential diagnosis of keloid and hypertrophic scar. Am J Dermatopathol. 2004;26(5):379-384.

126. Chipev CC, Simman R, Hatch G, Katz AE, Siegel DM, Simon M. Myofibroblast phenotype and apoptosis in keloid and palmar fibroblasts in vitro. Cell Death Differ. 2000;7(2):166-176.

127. Fujiwara M, Muragaki Y, Ooshima A. Keloidderived fibroblasts show increased secretion of factors involved in collagen turnover and depend on matrix metalloproteinase for migration. $\mathrm{Br} \mathrm{J}$ Dermatol. 2005;153(2):295-300.

128. Karouzakis E, Gay RE, Gay S, Neidhart M. Epigenetic control in rheumatoid arthritis synovial fibroblasts. Nat Rev Rheumatol. 2009;5(5):266-272.

129. Wang Y, Fan PS, Kahaleh B. Association between enhanced type I collagen expression and epigenetic repression of the FLI1 gene in scleroderma fibroblasts. Arthritis Rheum. 2006;54(7):2271-2279.

130. Russell SB, et al. Epigenetically altered wound healing in keloid fibroblasts. J Invest Dermatol. 2010;130(10):2489-2496

131. Li M, et al. Emergence of fibroblasts with a proinflammatory epigenetically altered phenotype in severe hypoxic pulmonary hypertension. J Immunol. 2011;187(5):2711-2722.

132. Beacham DA, Cukierman E. Stromagenesis: the changing face of fibroblastic microenvironments during tumor progression. Semin Cancer Biol. 2005;15(5):329-341.

133. Costea DE, et al. Identification of two distinct carcinoma-associated fibroblast subtypes with differential tumor-promoting abilities in oral squamous cell carcinoma. Cancer Res.
2013;73(13):3888-3901.

134. Gilchrest BA. A review of skin ageing and its medical therapy. Br J Dermatol. 1996;135(6):867-875.

135. El-Domyati $\mathrm{M}$, et al. Intrinsic aging vs. photoaging: a comparative histopathological, immunohistochemical, and ultrastructural study of skin Exp Dermatol.2002;11(5):398-405.

136. Mine S, Fortunel NO, Pageon H, Asselineau D. Aging alters functionally human dermal papillary fibroblasts but not reticular fibroblasts: a new view of skin morphogenesis and aging. PLoS One. 2008;3(12):e4066

137. Grillo HC. Origin of fibroblasts in wound healing. An autoradiographic study of inhibition of cellular proliferation by local x-irradiation. Ann Surg. 1963;157:453-467.

138. Boyce ST, Warden GD. Principles and practices for treatment of cutaneous wounds with cultured skin substitutes. Am J Surg. 2002;183(4):445-456.

139. Kirsner RS, Marston WA, Snyder RJ, Lee TD, Cargill DI, Slade HB. Spray-applied cell therapy with human allogeneic fibroblasts and keratinocytes for the treatment of chronic venous leg ulcers: a phase 2, multicentre, double-blind, randomised, placebo-controlled trial. Lancet. 2012;380(9846):977-985.

140.Wong T, et al. Potential of fibroblast cell therapy for recessive dystrophic epidermolysis bullosa. J Invest Dermatol. 2008;128(9):2179-2189.

141. Sorrell JM, Baber MA, Caplan AI. Human dermal fibroblast subpopulations; differential interactions with vascular endothelial cells in coculture: nonsoluble factors in the extracellular matrix influence interactions. Wound Repair Regen. 2008;16(2):300-309.

142.Walmsley GG, et al. Live fibroblast harvest reveals surface marker shift in vitro. Tissue Eng Part C Methods. 2015;21(3):314-321.

143. Watt FM. Mammalian skin cell biology: at the interface between laboratory and clinic. Science. 2014;346(6212):937-940. 